\title{
Analysis of Cost-Effectiveness of Trametinib Plus Dabrafenib as First-Line Therapy for Metastatic BRAFV600-Mutated Melanoma in China
}

\section{Guojun Sun}

Zhejiang University of Technology

Jingwen Wang

Zhejiang University of Technology

Xiaoying Zhou

Zhejiang University of Technology

Zhichao Hu

Zhejiang University of Technology

Zuojun Dong ( $\boldsymbol{\sim}$ jzd1970@zjut.edu.cn )

Zhejiang University of Technology

\section{Research Article}

Keywords: cost-effectiveness analysis, metastatic BRAF V600-mutated melanoma, dabrafenib, trametinib, vemurafenib

Posted Date: October 1st, 2021

DOI: https://doi.org/10.21203/rs.3.rs-941944/v1

License: (c) (i) This work is licensed under a Creative Commons Attribution 4.0 International License. Read Full License 


\section{Abstract}

Background: Treatment with trametinib plus dabrafenib for patients exhibiting metastatic BRAF V600mutated melanoma has been approved in China.

Method: We developed a Markov model to evaluate and compare the cost-effectiveness of trametinib plus dabrafenib against vemurafenib. Information on clinical situations, the rate of adverse reactions, follow-up treatments, and estimated transition probabilities were derived from the results of a clinical trial that compared treatment with trametinib plus dabrafenib against vemurafenib alone. A one-way sensitivity analysis and a probabilistic sensitivity analysis were conducted to assess the influence of uncertainty on the key model.

Result: Treatment with trametinib plus dabrafenib for one patient in the treatment period was estimated to cost CNY 332 294, and yield a total gain of 16.6 quality-adjusted life years (QALYs). Compared with vemurafenib, treatment involving trametinib plus dabrafenib yielded additional 3.96 QALYs, resulting in a unit cost-effectiveness of CNY 27460 per QALY. Sensitivity analysis shows that the results are reliable.

Conclusion囚From the perspective of China's health system, applying China's per-capita GDP in 2020 as the threshold of willingness-to-pay, it is cost-effective to treat metastatic melanoma patients exhibiting $B R A F \mathrm{~V} 600$ mutation with dabrafenib plus trametinib.

\section{Introduction}

Melanoma is a malignant tumor of melanocyte origin, characterized by high malignancy, high metastasis rate, high mortality, and poor prognosis. The incidence of melanoma is not high in China, but it has been on the rise in recent years. The number of new melanoma cases in 2020 around the world was 324635 , and the number of deaths was 57 043. About 6700 new melanoma cases and up to 3200 deaths are reported in China every year. ${ }^{1-3}$ Cutaneous melanoma is the predominant subtype in the west, but $66 \%$ of melanomas in Asia are limbal (43\%) and mucosal (23\%), which account for less than $5 \%$ of the subtypes in the West. ${ }^{4}$ Early-stage melanoma can be treated by surgical excision, but because of the differences in growth location and uneven prevalence, the Asian melanoma population is more likely to be in the middle to late stages at the time of diagnosis, with a five-year survival rate of only $5-10 \%{ }^{5}$

Most melanoma patients have mutations of the BRAF gene in the MAPK signaling pathway, often accompanied by the activation of targets such as B-Raf and MEK, so targeted therapy is one of the important strategies for treating metastatic melanoma with $B R A F$ mutations. ${ }^{6-8}$ About a quarter of melanoma patients in China have BRAF mutations, which often indicate worse clinical outcomes. ${ }^{9} \mathrm{~B}$-Raf is a serine/threonine kinase, and $B R A F$ mutations can regulate cell proliferation and survival through $\mathrm{B}$ Raf phosphorylation and the activation of MEK proteins (MEK1 and MEK2), thereby causing the activation of the downstream MAPK kinase pathway. BRAF mutations may therefore be considered a driver of cancer cell growth. ${ }^{10-11}$ In the past few years, drugs have been developed to inhibit the 
downstream mitogen-activated protein kinase (MAPK) cascade in melanoma cells. These targeted therapies have had a substantial clinical impact on melanoma patients with $B R A F$ mutations.

Vemurafenib is the first small molecule inhibitor of B-Raf approved by the FDA for the treatment of unresectable or metastatic melanoma, ${ }^{12-14}$ and the NMPA approved the marketing of vemurafenib in 2017. Clinical studies have shown that in melanoma patients with BRAFV600 mutation, vemurafenib improved overall and progression-free survival rates. Although vemurafenib monotherapy has achieved good results in comparison to chemotherapy, ${ }^{15-16}$ there are some problems, such as secondary resistance in $50 \%$ of the patients, which may be related to the reactivation of the MAPK pathway or the activation of bypass signaling pathways (e.g., PI3K/AKT/mTOR). ${ }^{17}$ MEK-a downstream protein of B-Raf -and B-Raf inhibitors in combination with MEK inhibitors may delay resistance and reduce adverse effects. In a large phase III randomized controlled trial (RCT), ${ }^{18}$ trametinib plus dabrafenib (TRAM+DAB) combination therapy was compared with vemurafenib (VEM) monotherapy. In patients with BRAF V600mutated melanoma, compared to VEM monotherapy-progression-free survival (PFS) of 7.3 months and overall survival (OS) of 17.2 months-the TRAM+DAB combination therapy significantly improved the PFS (11.4 months) and OS (median OS not reached), and overall toxicity was not increased.

The cost-effectiveness of the TRAM+DAB strategy and VEM monotherapy for melanoma in China has not previously been evaluated in studies. The aim of this research was to assess the cost-effectiveness of VEM monotherapy versus the TRAM+DAB strategy as first-line treatment for patients with metastatic unresectable melanoma in China, based on direct evidence provided in the clinical trial of Robert et al. ${ }^{18}$

\section{Methods}

\section{Study Population}

We used data from a published phase III RCT of patients with V600-mutated melanoma, in which the patients were randomized to receive as first-line treatment either trametinib plus dabrafenib or vemurafenib alone. ${ }^{18}$ In this trial, the median follow-up time of the TRAM+DAB combination treatment group and the VEM monotherapy group were 11 months and 10 months, respectively. The median age of the patients was 55 years for the TRAM+DAB treatment and 54 years for the VEM monotherapy.

\section{Markov Model}

A Markov model was established using TreeAge Pro 2011 to estimate the clinical and economic outcomes of vemurafenib and of trametinib plus dabrafenib. The Markov model consists of three states: PFS, progressive disease (PD), and death (D; Figure 1). Cycle lengths were 4 weeks, and the time horizon was lifetime. All patients were in the PFS state initially and could experience PD or D. They were randomized to receive either vemurafenib or trametinib plus dabrafenib orally until disease progression. Some patients chose to continue taking the study medication after the disease progression. 
The primary model outcomes were the utility and cost of the two treatment modalities, with utility expressed in quality-adjusted life years (QALYs), while the ICER was used to express the cost per QALY gained. Effects on the incremental cost-effectiveness ratio (ICER) were assessed, with discounting for costs and effects at $5 \%$ per year after the first year. Combining state values (e.g., life years, health-related quality of life, and cost) with the average number of cycles a patient stays in each state, it is possible to estimate life expectancy, life expectancy after quality adjustment, and expected costs. ${ }^{19}$ According to the National Bureau of Statistics of China, China's GDP per capita for 2020 was CNY 72 447; the willingnessto-pay (WTP) threshold for this study was set as CNY 72 447/ QALY.

\section{Model Inputs}

Inputs in our Markov model were 1) transition probabilities for PFS, PD, and D in each cycle; 2) probability of adverse effects under each treatment; 3 ) the utility of PFS and PD status under the two treatment regimens; 4) cost. From the two treatment regimens, PFS and OS data, incidence of adverse events, and transition probabilities were calculated from the clinical trial results of Robert et al. ${ }^{18}$ All parameter inputs are shown in Table 1.

\section{Cost \& Utility}

This analysis was from the perspective of China's health system, so only direct medical costs were considered in the model, including the costs of drugs, second-line treatment, general physical examinations, tumor assessment examinations, and the management of adverse reactions. The prices of vemurafenib, trametinib and dabrafenib were derived from the centralized drug procurement platform of Yunnan Province. Values for hematological examinations, CT scans, and other expenses came from public medical institutions. Only adverse reactions above grade 3 were considered; for vemurafenib treatment these were hypertension, arthralgia, skin rash, and increased levels of alanine aminotransferase and aspartate aminotransferase. For trametinib and dabrafenib treatment, the adverse reactions were fever and hypertension; these adverse reactions are generally alleviated after short treatment, and there are fewer cases of drug withdrawal. Therefore, in our Markov model, only the adverse-reaction management costs were included in the first-cycle cost calculation.

In the clinical trials, the researchers did not investigate the health-utility value of the patients in the PFS and PD stages; accordingly, the health-state utilities used in the model were obtained from the literature. ${ }^{20-22}$

\section{Sensitivity analyses}

Due to the uncertainty of parameters and assumptions, we conducted a one-way sensitivity analysis and probabilistic sensitivity analysis (PSA) to evaluate the robustness of the base-case results. The result of 
one-way sensitivity analysis is shown in Figure 2.

The PSA further explored the uncertainty of input parameters by randomly sampling the parameters (Table 1). Monte Carlo simulations were repeated over 1000 iterations to generate a distribution of ICER outcomes shown as a scatterplot (Figure 3). The cost-effectiveness acceptability curves showed the probabilities of each treatment being cost-effective under a wide range of WTP thresholds (Figure 4).

\section{Results}

The Markov model simulation results of this study were well matched to the data reported from the clinical trials. The median PFS time for TRAM+DAB in the study ${ }^{18}$ was 11.4 months, and 11.18 months based on the model. For vemurafenib, the PFS times were 7.3 months in the original study and 7.31 months in the model. In both cases, modelled results agree closely with the original research results. The model-based median OS of the vemurafenib group also matched well to the original data (17.6 months in the model and 17.2 months in the original data). For the TRAM+DAB strategy, the median oS had not been reached at the time of data extraction for publication.

From the result of the base-case model, TRAM+DAB treatment (total cost CNY 332 294; total effect 16.6 QALYs) compared with VEM treatment (total cost CNY 223 456; total effect 12.7 QALYs) leads to a gain of 3.9 QALYs per patient at an additional cost of CNY 108 838. This resulted in an ICER of CNY 27460 per QALY gained (Table 2). Discounting by $5 \%$ per year (starting after the first year) for costs and effects marginally increased the ICER.

One-way sensitivity analyses showed that varying the utility in the progression for the TRAM+DAB strategy had the highest impact on the ICER, followed by varying the utility in the PFS for the TRAM+DAB strategy. Further parameters that had a substantial impact were the costs of vemurafenib, dabrafenib, and trametinib, the utility value for patients in the VEM treatment in PD, and the utility value in PFS. When each parameter in the one-way sensitivity analysis was changed, the final results were not affected, proving that the conclusions obtained through the Markov model were reliable. In the analysis of the PSA results, the China's GDP per capita in 2020 was chosen as the WTP threshold according to the recommendations of the Chinese pharmacoeconomic evaluation guidelines; this was also considered suitable for the actual situations associated with the topic of this paper.

Probabilistic sensitivity analysis confirmed that the TRAM+DAB treatment is cost-effective. From the scatterplot in Figure 3 , it can be seen that $100 \%$ of the 1000 PSA iterations fall on the lower side of the WTP threshold. In the cost-effectiveness acceptability curve in Figure 4, the probability that the TRAM+DAB combination is economical at a WTP threshold of CNY 72774 is close to $100 \%$.

\section{Discussion}

The incidence of melanoma in China is increasing, and the development of diagnostic tests that detect specific mutations for directing targeted therapies has promoted targeted-therapy development. ${ }^{23-25}$ 
Previously, there have been no studies comparing the Chinese-context economics of dabrafenib combined with trametinib or vemurafenib in the treatment of patients with metastatic BRAFV600mutated melanoma. Based on data from the RCT, ${ }^{18}$ we developed a Markov model to evaluate vemurafenib treatment against the dabrafenib plus trametinib strategy, from the perspective of the Chinese health system. To adapt the model to better match the actual course of treatment for patients, the medical costs of second-line treatment after patient progression were included in the calculation of the costs. The results of the research found that TRAM+DAB therapy, compared against VEM monotherapy, resulted in an additional ICER of CNY 27460 per QALY gained, considering a discount rate of $5 \%$. This was well below the WTP threshold (CNY 72447 per QALY), indicating that the therapy was cost-effective. The uncertainty factors of the model were considered at all stages, and the results of the sensitivity analyses (both one-way sensitivity analysis and probabilistic sensitivity analysis) demonstrated the reliability of the model analysis results.

The BRAFi+MEKi dual-target regimen is recommended as a first-line treatment for patients with metastatic or unresectable melanoma with $B R A F$ mutations in the latest recommended clinical guidelines for melanoma from the Chinese Society of Anticancer Clinical Oncology Collaborative Center. Trametinib plus dabrafenib is currently the only approved BRAFi+MEKi dual-target regimen in China, and the results of this study demonstrate that TRAM+DAB combination therapy significantly improved overall patient survival with no increase in toxicity. Even though their costs have decreased substantially after multiple negotiations by the Nation Healthcare Security Administration of China-especially those of dabrafenib and trametinib, which were added to the medical insurance negotiation list shortly after their approval in China in 2019-their prices are still high for patients and the financial burden remains significant.

There are several limitations in our study. First, due to the lack of clinical trial data comparing the combination of trametinib and dabrafenib against vemurafenib alone in Chinese patients with metastatic BRAF V600-mutated melanoma, this study obtained patient survival data from a large phase III RCT conducted by Robert et al. ${ }^{18}$ However, the trial was primarily aimed at the Caucasian population. Second, at the time point of analysis as reported in the underlying trial by Robert et al., the median OS in the TRAM+DAB branch of the study had not yet been reached. Some patients may not have shown a longterm response, and the issue of such a long-term response may affect the ICER. Furthermore, because patients in the TRAM+DAB treatment group have a longer OS, it may lead to a higher cost, thereby reducing the cost-effectiveness of the treatment. Third, because of the lack of data on the utility of combination therapy, we used the utility for patients treated with trametinib monotherapy, and it is unclear what impact the combination therapy may have. As shown in the one-way sensitivity analyses, variation of utility parameters had a strong effect on the ICER, so using the utility of trametinib monotherapy instead of combination therapy might affect the ICER. Moreover, the model did not include utility for adverse effects from either treatment route, resulting in a higher utility for the DP status, potentially reducing disease burden. ${ }^{26}$ Fourth, as ipilimumab used in the systemic anticancer therapy was not available in China at the time, we instead used its launch price in Japan. 
In conclusion, although the findings in this paper show that trametinib in combination with dabrafenib therapy is cost-effective compared with vemurafenib monotherapy, both combination therapy and monotherapy are very expensive, with a high disease burden for patients, and further price reductions are needed to reduce the disease burden for patients.

\section{Declarations}

\section{Competing interests}

The authors declare that they have no competing interests.

\section{Funding}

This study was supported by grants from Zhejiang Federation of Humanities and Social Sciences (Grant number: Z20140118 ) and Science and Technology Department of Zhejiang Province( Grant number: KYY-ZX-20200202).

\section{Author Contributions}

Zuojun Dong: Conceived and designed the experiments. Xiaoying Zhou: Data collection, data analysis, and writing. Jingwen Wang: Data collection. Zhichao Hu: Data analysis. Guojun Sun: Revise.

Guojun Sun and Xiaoying Zhou are the Co-first authors.

\section{Ethical approval:}

This economic study is based on a literature review and model and does not require the approval of the institutional research ethics committee.

\section{Consent for publication}

Not applicable.

\section{Data availability:}

The datasets generated and analysed during the current study are available in the PubMed repository, https://pubmed.ncbi.nlm.nih.gov/25399551/. ${ }^{18}$

\section{Acknowledgement}

We would like to extend our special thanks to Zhejiang Federation of Humanities and Social Sciences, Science and Technology Department of Zhejiang Province.

\section{References}


1. Sung H, Ferlay J, Siegel RL, et al. Global Cancer Statistics 2020: GLOBOCAN Estimates of Incidence and Mortality Worldwide for 36 Cancers in 185 Countries. CA Cancer J Clin. 2021;71(3):209-249.

2. Schadendorf D, van Akkooi ACJ, Berking $C$, et al. Melanoma [published correction appears in Lancet. 2019 Feb 23;393(10173):746]. Lancet. 2018;392(10151):971-984.

3. Wanqing, Chen, Kexin, et al. Cancer incidence and mortality in China, 2014. Chinese Journal of Cancer Research. 2018(1):1-12.

4. Chi Z, Li S, Sheng X, et al. Clinical presentation, histology, and prognoses of malignant melanoma in ethnic Chinese: a study of 522 consecutive cases. BMC Cancer. 2011;11:85. Published 2011 Feb 25.

5. Sun L, Guan Z, Wei S, Tan R, Li P, Yan L. Identification of Long Non-coding and Messenger RNAs Differentially Expressed Between Primary and Metastatic Melanoma. Front Genet. 2019;10:292. Published 2019 Apr 5.

6. Mena E, Sanli Y, Marcus C, Subramaniam RM. Precision Medicine and PET/Computed Tomography in Melanoma. PET Clin. 2017 Oct;12(4):449-458.

7. Shahid K, Khalife M, Dabney R, Phan AT. Immunotherapy and targeted therapy-the new roadmap in cancer treatment. Ann TransI Med. 2019;7(20):595.

8. Zhou AY, Johnson DB. Combinatorial Therapies in Melanoma: MAPK Inhibitors and Beyond. Am J Clin Dermatol. 2018 Apr;19(2):181-193.

9. Bai X, Kong Y, Chi Z, et al. MAPK Pathway and TERT Promoter Gene Mutation Pattern and Its Prognostic Value in Melanoma Patients: A Retrospective Study of 2,793 Cases. Clin Cancer Res. 2017;23(20):6120-6127.

10. Maertens O, Kuzmickas R, Manchester HE, et al. MAPK Pathway Suppression Unmasks Latent DNA Repair Defects and Confers a Chemical Synthetic Vulnerability in BRAF-, NRAS-, and NF1-Mutant Melanomas. Cancer Discov. 2019;9(4):526-545.

11. Amaral T, Nouri N, Garbe C. The safety and efficacy of cobimetinib for the treatment of BRAF V600E or V600K melanoma. Expert Rev Anticancer Ther. 2016 Jul;16(7):705-15.

12. Ballantyne AD, Garnock-Jones KP. Dabrafenib: first global approval. Drugs. 2013 Aug;73(12):136776.

13. Hauschild A, Grob JJ, Demidov LV, et al. Dabrafenib in BRAF-mutated metastatic melanoma: a multicentre, open-label, phase 3 randomised controlled trial. Lancet. 2012;380(9839):358-365. doi:10.1016/S0140-6736(12)60868-X

14. Latimer NR, Abrams KR, Amonkar MM, Stapelkamp C, Swann RS. Adjusting for the Confounding Effects of Treatment Switching-The BREAK-3 Trial: Dabrafenib Versus Dacarbazine. Oncologist. 2015 Jul;20(7):798-805.

15. McArthur GA, Chapman PB, Robert C, et al. Safety and efficacy of vemurafenib in BRAF(V600E) and BRAF(V600K) mutation-positive melanoma (BRIM-3): extended follow-up of a phase 3, randomised, open-label study. Lancet Oncol. 2014;15(3):323-332. 
16. Chapman PB, Robert C, Larkin J, et al. Vemurafenib in patients with BRAFV600 mutation-positive metastatic melanoma: final overall survival results of the randomized BRIM-3 study. Ann Oncol. 2017;28(10):2581-2587.

17. Chapman $P B$, Hauschild A, Robert $C$, et al. Improved survival with vemurafenib in melanoma with BRAF V600E mutation. N Engl J Med. 2011;364(26):2507-2516.

18. Robert C, Karaszewska B, Schachter J, et al. Improved overall survival in melanoma with combined dabrafenib and trametinib. N Engl J Med. 2015;372(1):30-39.

19. Siebert U, Alagoz O, Bayoumi AM, et al. State-transition modeling: a report of the ISPOR-SMDM Modeling Good Research Practices Task Force--3. Value Health. 2012;15(6):812-820.

20. Amdahl J, Wang A, Thabane M, et al. Cost effectiveness of trametinib as first-line (1 I) treatment for Braf V600 positive advanced or metastatic melanoma - a Canadian societal perspective. Value Health 2014; 17:A83.

21. Schadendorf D, Amonkar MM, Milhem M, et al. Functional and symptom impact of trametinib versus chemotherapy in BRAF V600E advanced or metastatic melanoma: quality-of-life analyses of the METRIC study. Ann Oncol. 2014;25(3):700-706.

22. Beusterien KM, Szabo SM, Kotapati S, et al. Societal preference values for advanced melanoma health states in the United Kingdom and Australia. Br J Cancer. 2009;101(3):387-389.

23. Menzies AM, Long GV. Dabrafenib and trametinib, alone and in combination for BRAF-mutant metastatic melanoma. Clin Cancer Res. 2014;20(8):2035-2043.

24. Beale S, Dickson R, Bagust A, et al. Vemurafenib for the treatment of locally advanced or metastatic BRAF V600 mutation-positive malignant melanoma: a NICE single technology appraisal.

Pharmacoeconomics. 2013;31(12):1121-1129.

25. Aris M, Barrio MM. Combining immunotherapy with oncogene-targeted therapy: a new road for melanoma treatment. Front Immunol. 2015;6:46. Published 2015 Feb 9.

26. Matter-Walstra K, Braun R, Kolb C, et al. A cost-effectiveness analysis of trametinib plus dabrafenib as first-line therapy for metastatic BRAF V600-mutated melanoma in the Swiss setting. Br J Dermatol. 2015;173(6):1462-1470.

\section{Tables}

Table 1 Model input parameters, distribution, and range 


\begin{tabular}{|c|c|c|c|c|}
\hline \multirow[t]{2}{*}{ Patameter } & \multirow[t]{2}{*}{$\begin{array}{l}\text { Base } \\
\text { case }\end{array}$} & \multicolumn{2}{|l|}{ Range } & \multirow[t]{2}{*}{ Source } \\
\hline & & Low & High & \\
\hline \multicolumn{5}{|l|}{ DAB plus TRAM } \\
\hline \multicolumn{5}{|l|}{ 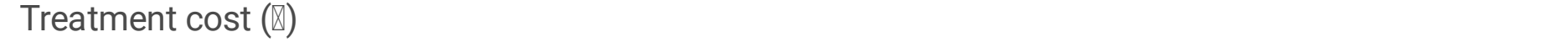 } \\
\hline DAB(150 mg orally twice daily,monthly (cost) & 10346.6 & 9311.9 & 11381.2 & $\begin{array}{l}\text { local } \\
\text { charget }\end{array}$ \\
\hline TRAM(2 mg orally once daily,monthly (cost) & 10346.0 & 9311.4 & 11380.6 & $\begin{array}{l}\text { local } \\
\text { charget }\end{array}$ \\
\hline Cost of managing adverse events $(\mathbb{\nabla})$ & 28.9 & 26.0 & 31.8 & $\begin{array}{l}\text { local } \\
\text { charget }\end{array}$ \\
\hline $\begin{array}{l}\text { Cost of Subsequent Systemic Anticancer Drug } \\
\text { Therapy in FU }\end{array}$ & 2278.5 & 2050.7 & 2506.4 & $\begin{array}{l}\text { local } \\
\text { charget }\end{array}$ \\
\hline 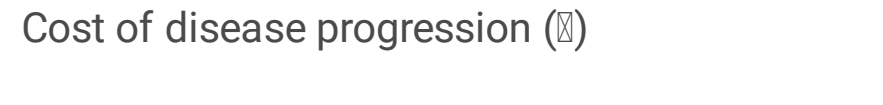 & 2519.4 & 2267.4 & 2771.3 & $\begin{array}{l}\text { local } \\
\text { charget }\end{array}$ \\
\hline 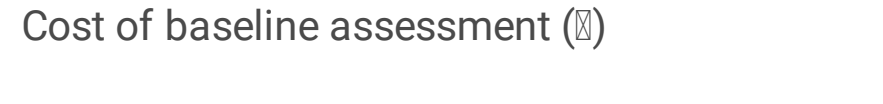 & 30.4 & 27.4 & 33.5 & $\begin{array}{l}\text { local } \\
\text { charget }\end{array}$ \\
\hline Cost of physical checkment ( () & 145.2 & 130.7 & 159.7 & $\begin{array}{l}\text { local } \\
\text { charget }\end{array}$ \\
\hline \multicolumn{5}{|l|}{ Health utilities } \\
\hline Utility progression-free survival & 0.78 & 0.70 & 0.86 & [20®21] \\
\hline Utility progression & 0.72 & 0.65 & 0.79 & [20ه21] \\
\hline \multicolumn{5}{|l|}{ VEM } \\
\hline \multicolumn{5}{|l|}{ Treatment cost $(\mathbb{\nabla})$} \\
\hline VEM(960 mg orally twice daily,monthly (cost) & 20090.6 & 18081.5 & 22099.6 & $\begin{array}{l}\text { local } \\
\text { charget }\end{array}$ \\
\hline Cost of managing adverse events $(\mathbb{\nabla})$ & 49.0 & 44.1 & 53.9 & $\begin{array}{l}\text { local } \\
\text { charget }\end{array}$ \\
\hline $\begin{array}{l}\text { Cost of Subsequent Systemic Anticancer Drug } \\
\text { Therapy in FU }\end{array}$ & 3638.3 & 3274.5 & 4002.2 & $\begin{array}{l}\text { local } \\
\text { charget }\end{array}$ \\
\hline Cost of disease progression $(\mathbb{\nabla})$ & 2476.7 & 2229.0 & 2724.3 & $\begin{array}{l}\text { local } \\
\text { charget }\end{array}$ \\
\hline Cost of baseline assessment ( $(\mathbb{)})$ & 30.4 & 27.4 & 33.5 & $\begin{array}{l}\text { local } \\
\text { charget }\end{array}$ \\
\hline Cost of physical checkment $(\mathbb{\nabla})$ & 145.2 & 130.7 & 159.7 & $\begin{array}{l}\text { local } \\
\text { charget }\end{array}$ \\
\hline
\end{tabular}


Health utilities

Utility progression-free survival

0.80

0.72

0.88

[22]

Utility progression

0.52

0.47

0.57

[22]

DAB dabrafenib; TRAM trametinib; VEM vemurafenib; FU follow-up; tThe price of drugs comes from the information disclosure of Yunnan drug centralized purchasing center

Table 2 Cost-effectiveness of trametinib plus dabrafenib and vemurafenib as first-line treatment of metastatic BRAF V600-mutated melanoma

\begin{tabular}{|c|c|c|c|c|c|}
\hline \multirow[t]{2}{*}{ Treatment } & \multirow[t]{2}{*}{ cost(吅 } & effectiveness & \multirow{2}{*}{$\begin{array}{l}\text { incremental } \\
\text { cost(D) }\end{array}$} & \multirow{2}{*}{$\begin{array}{l}\text { incremental } \\
\text { effectiveness(QALY) }\end{array}$} & ICER \\
\hline & & (QALY) & & & (प/QALY) \\
\hline VEM & 223456.26 & 12.65 & 0 & 0 & \\
\hline $\begin{array}{l}\text { TRAM } \\
\text { +DAB }\end{array}$ & 332294.57 & 16.61 & 108838.31 & 3.96 & 27460.87 \\
\hline
\end{tabular}

DAB, dabrafenib TRAM, trametinib; VEM vemurafenib; QALY quality-adjusted life year; ICER incremental cost-effectiveness rati

\section{Figures}

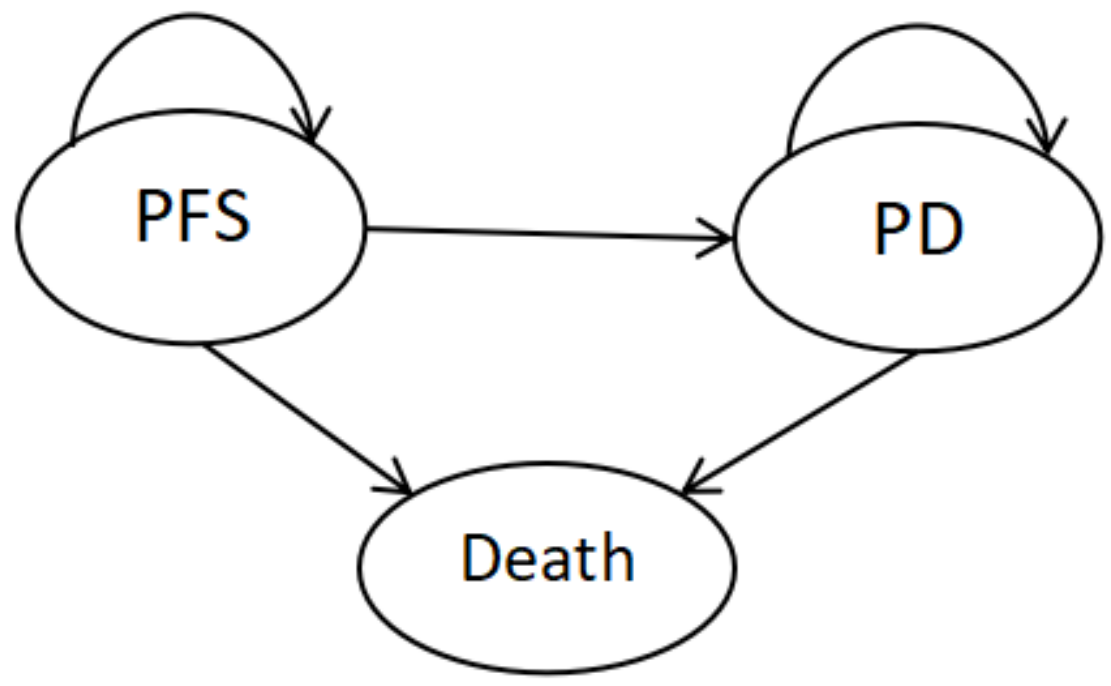

Figure 1 
Markov model for patients with previously untreated metastatic melanoma with BRAF V600E or V600K mutations

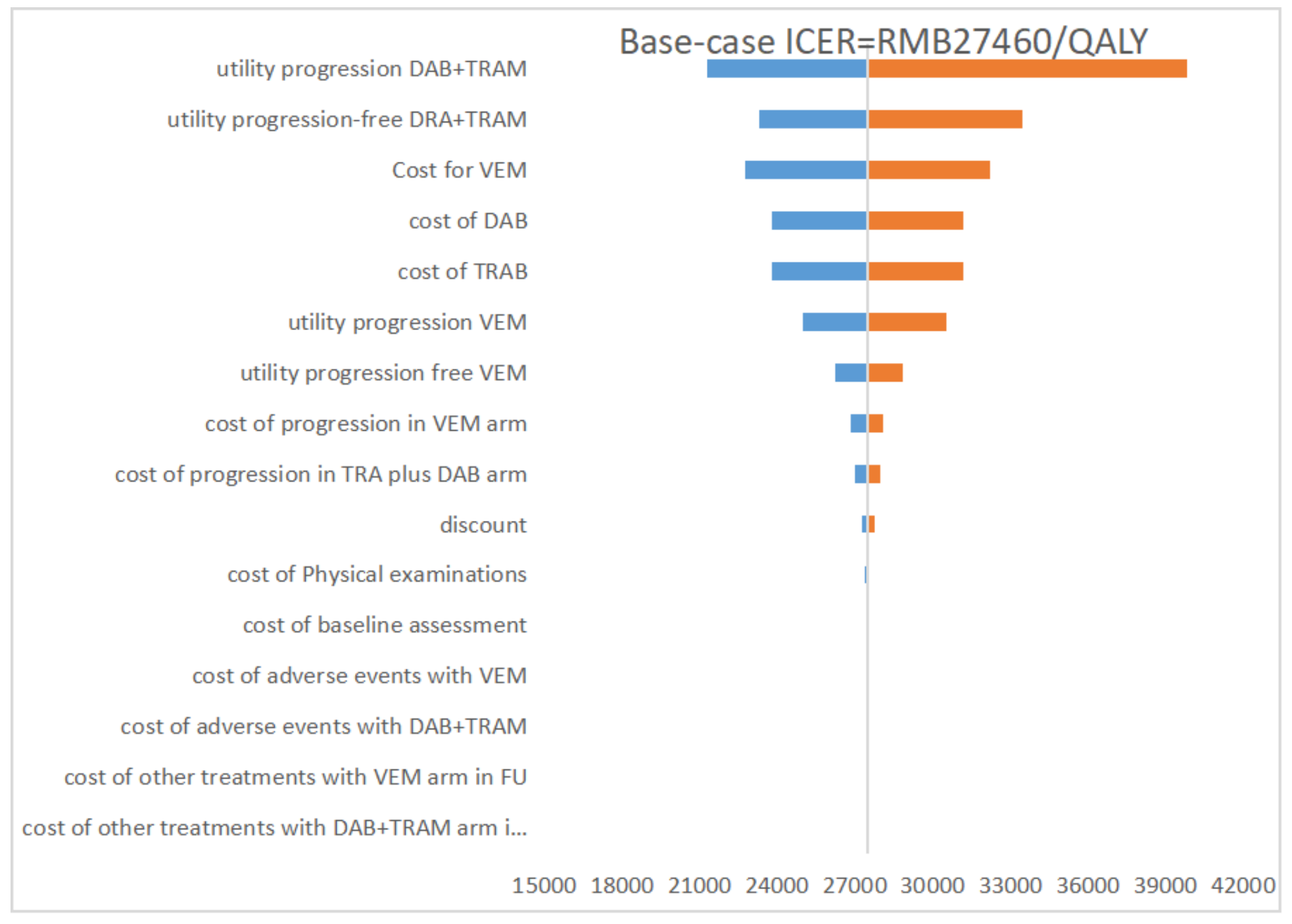

\section{Figure 2}

One-way sensitivity analysis based on the parameters as shown in Table 1. Results shown are for $5 \%$ discounting. DAB, Dabrafenib; TRAM, Trametinib; VEM, Vemurafenib; FU, follow-up; QALY, quality-adjusted life year. 


\section{Incremental Cost-Effectiveness, PLUS v. VEM}

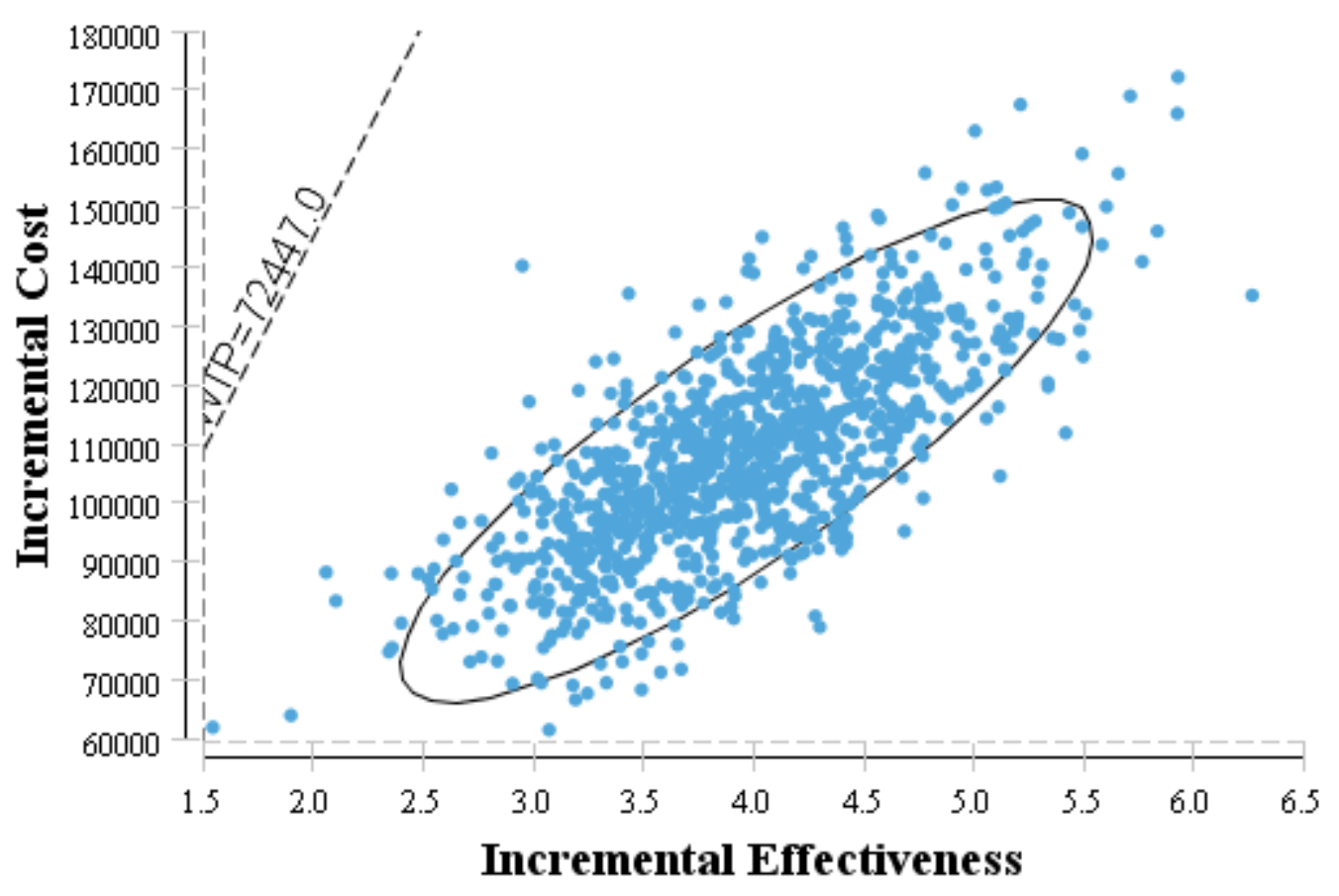

Figure 3

Probabilistic sensitivity analysis for trametinib plus dabrafenib or vemurafenib as first-line therapy for metastatic BRAF V600-mutated melanoma in china. willingness to pay (WTP) $=$ RMB72,447 per qualityadjusted life year (QALY). Results shown are for $5 \%$ discounting

\section{CE Acceptability Curve}

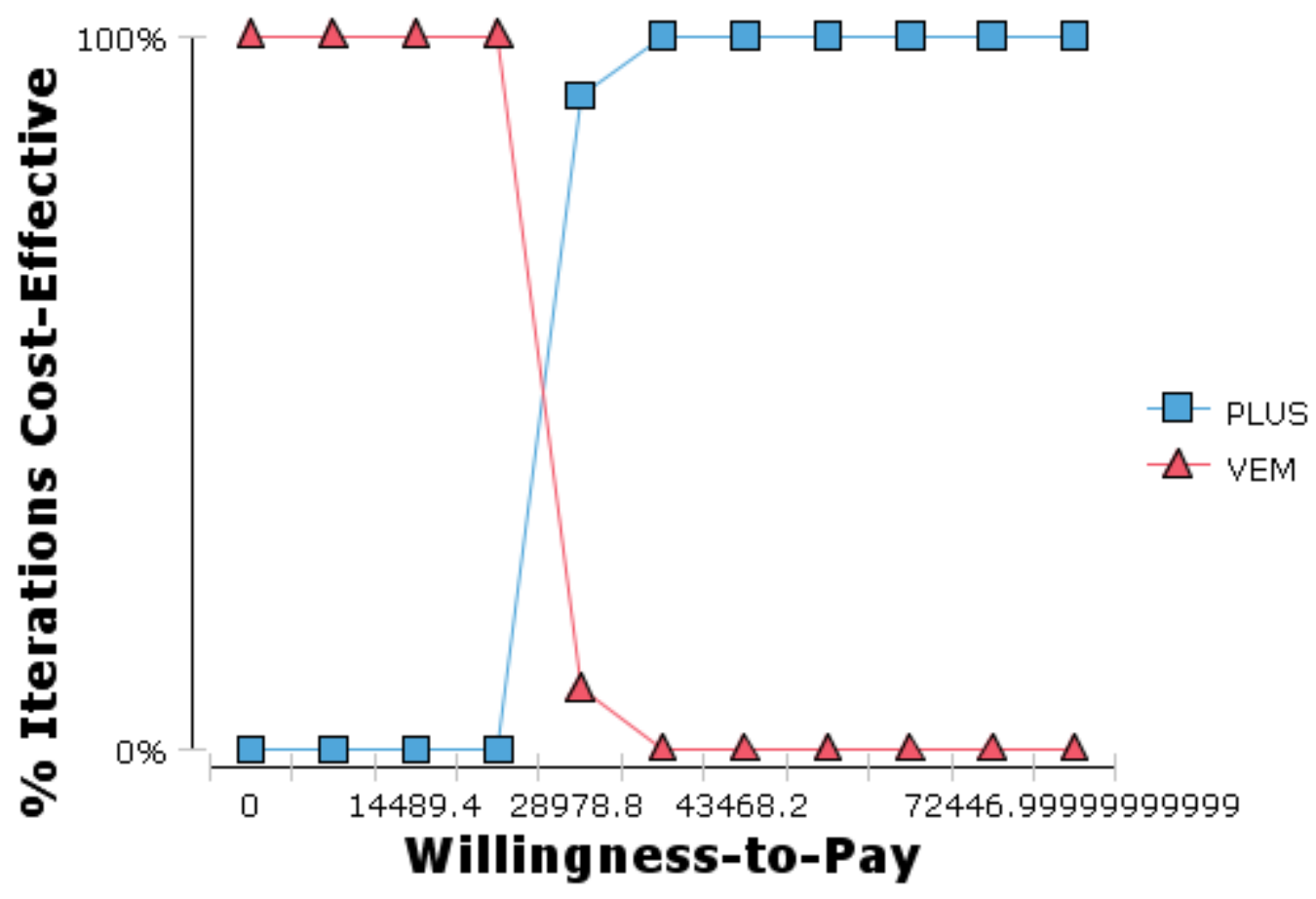


Figure 4

Cost-effectiveness acceptability curves; PLUS, trametinib plus dabrafenib; VEM, vemurafenib 